\title{
Pulmonary artery aneurysm in Behcet's disease: a ticking time bomb
}

\begin{abstract}
Behcet's disease (BD) is a rare condition in sub-Saharan Africa. It often manifests as recurrent oral and genital aphthous ulcers with ocular features and infrequently involving major systems like pulmonary vasculature and parenchyma. Pulmonary involvement in $\mathrm{BD}$, although rare, can manifest as pulmonary artery aneurysm, vascular thrombosis, pulmonary infarction, pneumonia and pleurisy.

Our index case is a 38 year old Nigerian who presented with recurrent orogenital ulcer, hemoptysis and uveitis. He had a contrast enhanced chest CT scan that revealed pulmonary artery aneurysm. His symptoms resolved following commencement of immunosuppressant and steroid.
\end{abstract}

Increased awareness is required for early detection of $\mathrm{BD}$ especially in regions where it is regarded as rare. Furthermore, multidisciplinary approach in its management is vital to reduce morbidity and mortality.

Keywords: behcet's disease, pulmonary vasculitis, pulmonary artery aneurysm, Nigeria
Volume 7 Issue 3 - 2017

\author{
Adeoti AO,' Ibidapo OR,' Ojo O² \\ 'Department of Medicine, Ekiti State University Teaching \\ Hospital, Nigeria \\ ${ }^{2}$ Rheumatology Unit, Kubwa General Hospital, Nigeria
}

\begin{abstract}
Correspondence: Adekunle Olatayo ADEOTI, Respiratory Unit, Department of Medicine, Ekiti State University Teaching Hospital, Nigeria, Email kadeoti2002@yahoo.com
\end{abstract}

Received: April 28, 2017| Published: September 07, 2017

\section{Introduction}

Behcet's disease is a rare multi systemic disorder of unknown etiology which often manifest with recurrent oral aphthous ulcers, genital ulcers and ocular lesions. It occasionally manifest as severe systemic presentations involving the pulmonary, cardiovascular system, central nervous system, and gastrointestinal system. ${ }^{1}$

Behcet's disease (BD) has a worldwide prevalence which ranges between $0.1 / 1000$ and 1/10,000 with a significant presence in Asian countries from the Mediterranean to Japan, hence the term Silk Road Disease. ${ }^{2}$ Turkey has reported the highest prevalence ranging from 80 to $420 / 100,000$. However, there are few reported cases in Africa, North Europe and America. ${ }^{3,4}$ Males in western Asia are commonly affected as opposed to females in Japan and Korea. The usual age at presentation is between the third and fourth decades. ${ }^{2}$

Although the underlying cause of BD is unknown, like other autoimmune disorders, it is postulated to occur in genetically predisposed individuals exposed to triggering agents like microbes and environmental factors. The presence of an HLA-B51 allele is associated with increased risk of developing this condition as well as worsened presentation. ${ }^{5,6}$

The clinical presentation is highly variable and no specific laboratory investigation is available for diagnosing BD. Several diagnostic criteria were proposed in the last 70years with different racial considerations but none is specific to individuals of African descent. However, two separate consensus criteria made by the collaboration of experts from several countries; International Study Group on Behcet's Disease (ISG) and International Criteria of Behcets Disease (ICBD), have received wide acceptance with varying sensitivity and specificity. ${ }^{7}$ The use of anti- inflammatory and immunosuppressive agents is important in relieving symptoms as no curative solution is currently available. ${ }^{8}$

\section{Case presentation}

A 38 year old Nigerian male who presented to the respiratory clinic with a history of chronic cough productive of blood stained sputum of 12 months duration, recurrent oral and genital ulcers of 12 months, low grade fever of 10 months and 8months history of progressive weight loss. Mouth ulcers were recurrent and painful, involving the tongue and gum. The genital ulcers were multiple and painful with no bleeding or discharges and healed spontaneously without scars. He had had about 5 recurrences in the last 12 months. There was associated history of blurring of vision and occasional redness of the eyes with no eye discharge. The patient reported no history of joint pains or swelling. He denied history of contact with adult with chronic cough or past treatment for pulmonary tuberculosis or any history suggestive of sexually transmitted infections in the past. He also denied use of sculpture containing drugs and occupational or environmental exposure to toxic agents. His other family members were healthy and none had history of similar illness. He recently got married but his wife is yet to conceive.

Physical examination revealed a wasted young man (BMI $18.1 \mathrm{~kg} /$ $\mathrm{m}^{2}$ ) who was afebrile with no lymphadenopathy. He had multiple tender oral ulcers measuring about $1-2 \mathrm{~mm}$ in diameter on the tongue and gum with an erythematous base. However, normal male genitalia with healed without scars and there was no oedema or tenderness of the testicles. Ophthalmologic examination revealed normal visual acuity and optic nerve with features of uveitis on slit lamp examination.

The results of laboratory investigation revealed packed cell volume $(38 \%)$. Other cells counts were within normal limit but for the peripheral blood film that showed microcytes, macrocytes and anisocytosis. Chest radiograph showed widespread rounded opacities in both lung fields while constrast enhanced CT scan revealed bilateral multiple saccular dilatations of the pulmonary arterials in keeping. Sputum for acid and alcohol fast bacilli and Gene expert (negative), erthrocyte sedimentation rate $(40 \mathrm{~mm} / \mathrm{hr})$, Hepatitis screening (HBsAg, Anti-HCV were both negative), HIV rapid serology test (negative), and VDRL for Syphilis (negative). The values of AntiNuclear Antibody, pANCA, cANCA were within normal limit and pathergy test was negative after $48 \mathrm{hrs}$. 
An assessment of Behcet's disease was made based on the presence of recurrent oral ulcer ( $>3$ times in a year), recurrent genital ulcers and posterior uveitis as proposed by International Study Group on Behcet Disease. He was commenced on oral prednisolone 10mg bid, oral azathioprine $50 \mathrm{mg}$ bid and omeprazole $20 \mathrm{mg}$ b.d. The dose of the oral prednisolone was tapered off after 2 weeks. He made significant clinical improvement as all symptoms resolved after 2 weeks of treatment and complete resolution of symptoms was noticed after a month (Figures 1-3).

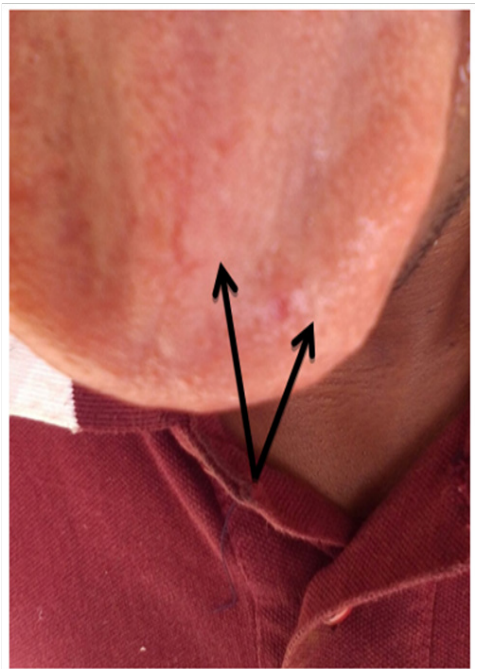

Figure I Black arrows indicate areas of multiple ulcers on the tongue.

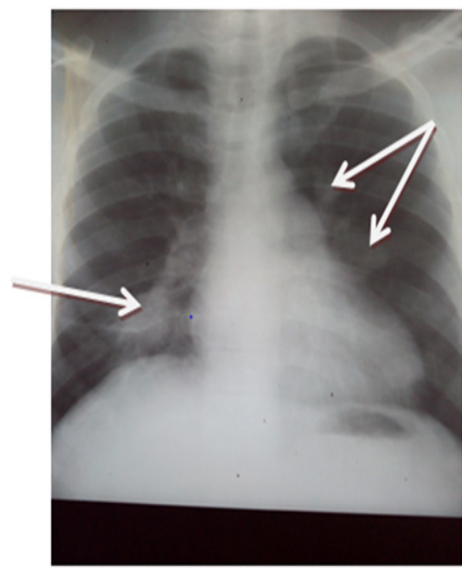

Figure 2 Chest X-ray: arrows show rounded opacities seen in both lung fields.

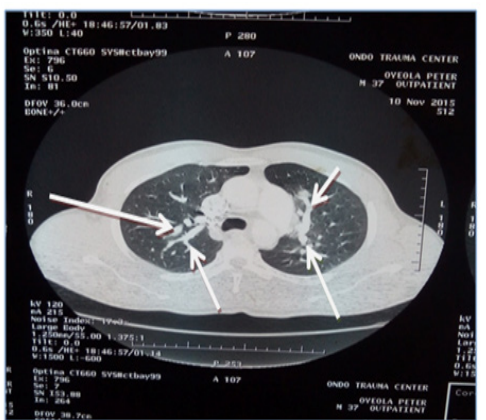

Figure 3 Shows a contrast enhanced Chest CT of patient that revealed bilateral multiple saccular dilatations of the pulmonary arterial branches.

\section{Discussion}

Behcet's Disease (BD) is a multi systemic disorder of unknown etiology, rarely reported in sub-Saharan Africa, characterized by recurrent attacks of oral aphthous ulcers, genital sores, and ocular manifestations. ${ }^{2,4}$ It runs a severe course in men and those with onset before 25years of age. ${ }^{1}$ Vascular involvement is the most common cause of mortality and associated with poor prognosis. Its pulmonary manifestations could mimic conditions like pulmonary tuberculosis, especially in tuberculosis high burden countries. ${ }^{9}$

Our index patient is a male in his fourth decade with recurrent orogenital ulcers and uveitis. This is in tandem with the diagnostic criteria for $\mathrm{BD}$ as well as the archetypal chest $\mathrm{CT}$ finding of pulmonary artery aneurysm. ${ }^{7,10}$ The age of onset in our patient is higher than the 25years associated with poor prognosis, however, the gender as well as the evidence of pulmonary artery aneurysm has been associated with high mortality and morbidity.

Pathergy test is the hyper-reactivity of the skin to intracutaneous injection or needle prick but this was negative in our patient. It is demonstrated more frequently in specific population with rare occurrence in African studies., ${ }^{411}$ This could be responsible for the negative pathergy test in our patient as HLA B51 influences clinical features of BD in susceptible individuals. ${ }^{12}$

ANCA- associated vasculitis that mostly affect the lungs were excluded as connective tissue disease serologies and anti-neutrophil cytoplasmic antibody (ANCA) were normal. Similarly, Hughesstovin syndrome (HSS), a close differential of BD with consistent radiologic and histopathologic finding was unlikely as our patient had both oral and genital ulcer which are usually absent in HSS. ${ }^{13}$ Patient could have been managed as smear negative tuberculosis in a high burden country but for the rapid and sensitive diagnostic test of Xpert MTB/RIF test which was also negative.

Our patient was commenced on immunosuppressive agents and steroid as advocated by the European League against Rheumatic Diseases (EULAR) and there was significant clinical improvement within 2 weeks with no side effects. Recommended treatment for pulmonary aneurysm is mainly with immunosuppressives as surgery is associated with high risk of mortality. Furthermore, patients with ocular involvement benefit from azathioprine and steroid to prevent irreversible damage and visual loss. ${ }^{14}$

\section{Conclusion}

It is apparent that the management of Behçet's disease may be challenging to the attending physicians, especially among populations with documented rare occurrence. There is, therefore, a need to increase the level of awareness about BD among physicians for prompt diagnosis and treatment of individuals with $\mathrm{BD}$ so as to prevent disability and reduce associated mortality.

\section{Consent}

All authors declare that written informed consent was obtained from the patient.

\section{Acknowledgements}

None. 


\section{Conflict of interests}

The author declares no conflict of interests.

\section{References}

1. Erkan F, Gül A, Tasali E. Pulmonary manifestations of Behçet's disease. Thorax. 2001;56(7):572-578.

2. Zeidan MJ, Saadoun D, Garrido M, et al. Behçet's disease physiopathology:a contemporary review. Autoimmunity Highlights. 2016;7(1):4.

3. Yazici H, Akokan G, Yalcin B, et al. The high prevalence of HLAB5 in Behçet's disease. Clinical and experimental immunology. 1977;30(2):259-261.

4. Ajose FO, Adelowo O, Oderinlo O. Clinical presentations of Behçet's disease among Nigerians: a 4-year prospective study. Int $j$ dermatol. 2015;54(8):889-897.

5. Sakane T, Takeno M, Suzuki N, et al. Behçet's disease. New England Journal of Medicine. 1999;341(17):1284-1291.

6. Choukri F, Chakib A, Himmich H, et al. HLA-B phenotype modifies the course of Behçet's disease in Moroccan patients. Tissue Antigens. 2003;61(1):92-96.

7. Davatchi F, Sadeghi Abdollahi B, Chams-Davatchi C, et al. The saga of diagnostic/classification criteria in Behcet's disease. Int $\mathrm{j}$ Rheum dis. 2015;18(6):594-605.
8. Alibaz-Oner F, Karadeniz A, Ylmaz S, et al. Behcet disease with vascular involvement: effects of different therapeutic regimens on the incidence of new relapses. Medicine. 2015;94(6):e494.

9. Sanyika C, Corr P, Royston D, et al. Pulmonary angiography and embolization for severe hemoptysis due to cavitary pulmonary tuberculosis. Cardiovasc and intervent radiol. 1999;22(6):457-460.

10. Celenk C, Celenk P, Akan H, Başoğlu A. Pulmonary artery aneurysms due to Behçet's disease: MR imaging and digital subtraction angiography findings. AJR American journal of roentgenology. 1999;172(3):844-845.

11. Ndiaye M, Sow AS, Valiollah A, et al. Behcet's disease in black skin. A retrospective study of 50 cases in Dakar. J Dermatol Case Rep. 2015;9(4):98-102.

12. Ryu HJ, Seo MR, Choi HJ, et al. Clinical phenotypes of Korean patients with Behcet disease according to gender, age at onset, and HLA-B51. Korean J Intern Med. 2018;33(5):1025-1031.

13. Ceylan N, Bayraktaroglu S, Erturk SM, et al. Pulmonary and vascular manifestations of Behcet disease: imaging findings. American Journal of Roentgenology. 2010;194(2):W158-W164.

14. Hatemi G, Silman A, Bang D, et al. EULAR recommendations for the management of Behçet disease. Ann rheum dis. 2008;67(12):1656-1662. 